\title{
W UMa Binaries
}

\author{
O. Vilhu \\ Observatory and Astrophysics Laboratory, University of Helsinki, SF-00130 \\ Helsinki, Finland
}

\begin{abstract}
The origin and evolution of contact (W UMa) binaries are discussed with the help of the braking law of eq.(1). Best observationally justified parameter-values in (1) for W UMa, CV and LMXB binaries are: $\alpha \approx 1.5, \gamma=2$ and $f$ computed from eq.(2). A semi-empirical relation (eq.(5)) between the scaled magnetic torque and the fractional $\mathrm{X}$ ray luminosity is proposed. First attempts of simultaneous photometric and spectroscopic surface imaging of contact binaries are described.
\end{abstract}

\section{Introduction}

Huang (1966) suggested that magnetic torques could bring together the separate components of a detached binary. This is Schatzman's (1962) and Mestel's (1968) mechanism of magnetic braking. Further work has concentrated on details of this evolution before and during the contact phase (Van't Veer, 1979; Rahunen, 1981; Vilhu, 1982; Rahunen and Vilhu, 1982; Rucinski, 1985; Guinan and Bradstreet, 1988; Hilditch, King and McFarlane, 1988; Van't Veer and Maceroni, 1989). Fig.1 shows the scenario in the period-colour diagram. The diagram itself, together with many other observational and theoretical properties of W UMa stars, are discussed in the excellent reviews by Rucinski (1985a,b).

In this picture, detached short period systems (1 - $4 \mathrm{~d})$ are progenitors of contact W UMa binaries. Contact models with gradual angular momentum loss (AML) evolve in the direction of the observed boundaries of the period-colour diagram (Rahunen, 1981; Rahunen and Vilhu, 1982). The mass ratio decreases (becomes more extreme) on the secondary's thermal time scale $310^{7} m^{-3.5}$ years, where $m$ is the mass in solar units. The nuclear evolutionary state of the primary determines the horizontal distance from the observed boundaries (see Fig.1). Zero age systems occupy the left boundary, whereas the evolved ones (non-homogeneous primary) are closer to the right border.

The models turn to the right in the period-colour diagram when the secondary's thermal time scale becomes shorter than the primary's nuclear time $10^{10} m^{-3.5}$ years. This happens around the mass ratio $q=0.2$. After this point the evolution is determined by the nuclear evolution of the primary. The final product is probably a 
single rapidly rotating star. This could be either a giant of FK Com type (Webbink, 1976; Bopp and Rucinski, 1981) or a single A-star (like blue stragglers in open clusters, Eggen and Iben, 1990; Mateo et al. 1990).

During the contact evolution the secondary's thermal structure strongly deviates from thermal equilibrium. Although the spectral types of the components are roughly equal, the secondary's convective envelope is much shallower than that of the primary. For this reason, we can expect that the primary has more magnetic activity on its surface; the dynamo works more efficiently with rapid rotation and deep convective zones. In principle, this can be observationally verified by the surface imaging techniques (see Ch.3).

The scenario predicts that the periods of $\mathrm{W}$ UMa stars should gradually increase by $d P / d t=+10^{-8} P /$ year. This is much less than the observed period changes (Yamasaki, 1975) which are sometimes positive, sometimes negative. These could be due to the effects of spots, the possible 3rd body, random changes in the mass exchange etc. It is difficult to separate the slow trend from all possible short time effects (Herczeg, 1991; Kim, 1991). For the detached system ER Vul, eq.(1) predicts $d P / d t=-10^{-9} P /$ year $(a=1.5)$.

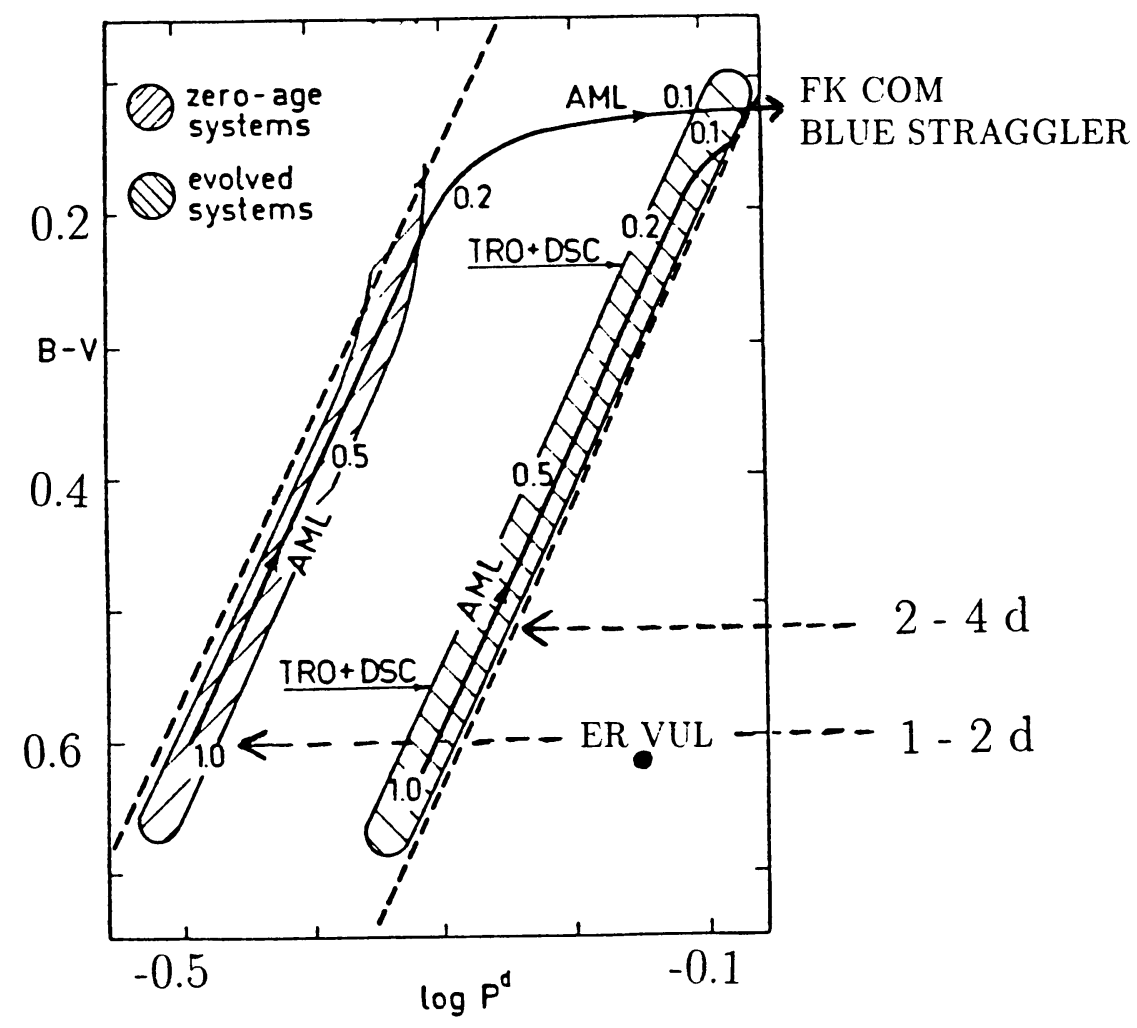

Fig.1 The period-colour diagram for contact binaries of W UMa type with the angular momentum loss scenario. 


\section{Magnetic Braking}

\subsection{Braking Laws}

A rather general form of the braking torque, used in the literature, is

$$
d J / d t=-1.010^{42} k^{2} f^{-2} m r^{\gamma} P_{3}^{-\alpha},
$$

in units of $\mathrm{g} \mathrm{cm}^{2} \mathrm{sec}^{-1}$ year $^{-1}$. In this formula the gyration constant $k^{2}$ is roughly 0.1 in main sequence stars. The period $P$ is expressed in units of 3 days, $m$ and $r$ are the mass and radius in solar units. When eq.(1) is applied to late type binary stars, it is assumed that there is an efficient coupling between the orbital and spin motions.

Eq. (1) has been widely applied to contact as well as to cataclysmic (CV) and low mass X-ray (LMXB) binaries. We list some possible parameter values used:

1. Verbunt and Zwaan (1981, VZ) used $f=0.73-1.78, \gamma=4$ and $\alpha=3$. VZ applied the formula to the cool companion to study the mass transfer of CV's and LMXB's. They quite succesfully explained the rate predicted by accretion luminosities.

2. Rappaport, Verbunt and Joss $(1983, \mathrm{RVJ})$ allowed the $\gamma$-value to change between 2 and 4 , and followed the $\mathrm{CV}$ evolution.

3. Vilhu (1982) used $f=1, \gamma=4$ and $\alpha=3$ for periods over 3 days and $\alpha=1.5$ for shorter ones. He applied eq.(1) to explain detached systems as progenitors of W UMa stars. The formula briefly explains the average space density of W UMa stars (= 1 percent of all stars of the same spectral type: Van't Veer, 1975; Fleming et al. 1989) and the duration of the contact phase ( $510^{8}$ years) derived from model computations. The large kinematical age of W UMa stars (8-10 $10^{9}$ years, Guinan and Bradstreet, 1988) can also be understood by this scenario.

4. The VZ-formula was originally derived from the Skumanich (1972) law for single solar type stars. However, this law can now be re-written, to include different masses $(m=0.6-1.4)$. Using the Catalano et al. (1988) results $\left(P=f^{-1} a g e^{0.5}\right)$, with some simple algebra, we find that $\gamma=2, \alpha=3$ and

$$
f=(3.3-2.3 m)^{-1},
$$

where $m$ is the mass in solar units. These are so far the best observationally verified parameter-values of eq.(1). However, the periods of W UMa's and CV's are short and outside the range of Catalano's sample. Therefore, one can still play with other parameter values, taking only care that they asymptotically approach the numbers above.

If $f$ is computed from (2) and $\gamma=2$ (instead of 4 of VZ), then $\alpha$ should be $=$ 1.6 , in order to get the same torque for CV's as computed by VZ. This value is in the same direction as obtained for W UMa's (1.5).

The size of the torque (eq.1) can be conventionally compared with the sizes of the spin and orbital angular momenta:

$$
\begin{gathered}
J_{\text {spin }}=2.3310^{50} k^{2} m r^{2} P_{3}^{-1} \\
J_{\text {orb }}=1.1410^{52} q(1+q)^{-2} m_{\text {tot }}^{5 / 3} P_{3}^{1 / 3}
\end{gathered}
$$




\subsection{Connection Between the Torque and the X-ray Flux}

Since the magnetic braking acts through the hot corona (Mestel and Spruit, 1987; Moss, 1986; Vilhu and Moss, 1986; Budding et al. 1991) one can expect a correlation between the braking torque and the X-ray luminosity.

Fig.2 shows the observed surface fluxes for different classes of cool stars (from Vilhu, 1987). In this plot the solid line (with an arrow) is drawn through the colours of the contact models by Rahunen (1981). The dashed oval shows the expected region occupied by the CV and LMXB secondaries.

The braking torques of W UMa's were estimated from Rahunen's (1981) models, these are close to those found from eq. (1) with $\alpha=1.5, \gamma=4$ and $f=1$ (using the primary). In Fig. 3 these are plotted against the fractional X-ray fluxes, estimated from Fig. 2. In a similar manner cool components of CV's can be placed in Fig.3. For $f=1, \alpha=3$ and $\gamma=4$ (VZ values) the scaled torque is independent of the secondary's mass (the lowest triangle in Fig.3). The two upper triangles are computed for $f=1, \alpha=3, \gamma=2$ (RVJ values) and for two masses ( 0.5 and 0.3 solar masses, corresponding to binary periods of 5 and 3 hours, respectively). Using $\alpha$ $=1.5$ and computing $f$ from (2), these last two values should be shifted down in Fig. 3 by 0.5 and 0.2 , respectively. Hence, the 'best' values $(\alpha=1.5, \gamma=2$ and $f$ from (2)) are pretty close to the VZ torque.

The short period detached binary ER Vulpecula $(\mathrm{G} 0+\mathrm{G} 5, \mathrm{P}=0.7 \mathrm{~d})$ is plotted in Fig.3 with the observed $L_{X} / L_{b o l}=10^{-3.2}$ and with the torque computed from (1), using $\alpha=1.5$ (the lower diamond) and $\mid$ alpha $=3$ (the upper diamond). The $\mathrm{G}$ stars in the Hyades, Pleiades and Orion clusters are plotted with their observed mean X-ray fluxes (Caillault and Helfand, 1985) and using the torque $d J / d t=-0.5 J_{s p i n} /$ age (which follows from $P=a(t+c)^{0.5}$ with solid body rotation (Catalano et al. 1988)).

The dashed line in Fig. 3 gives a linear fit:

$$
d J / d t=-J_{s p i n} 10^{-3.5} L_{X} / L_{b o l} .
$$

This is consistent with the well known approximative scaling law's at G2: $L_{X}=$ $a g e^{-1}$ (Caillault and Helfand, 1985), $L_{X}=v_{\text {rot }}^{2}$ (Pallavicini et al. 1981) and $P=$ age $e^{0.5}$ (Skumanich, 1972), up to some constant factors. However, the first of these is very crude, much better fit would be an exponential one.

\section{Surface Imaging of W UMa Stars}

\subsection{The Neck}

The neck is one of the most dramatic places in a contact binary. Through the neck the primary pushes large amounts of luminosity into the secondary. Using EXOSAT-observations, Vilhu and Heise (1986) explained the soft X-ray light curve of VW Cep with an active neck. Bradstreet and Guinan (1990) found extra $M g I I$ line emission, which can be interpreted as coming from the neck region. The emission can be seen at different epochs (see Fig.4), it is also visible in W UMa (Vilhu and Walter, 1987). 


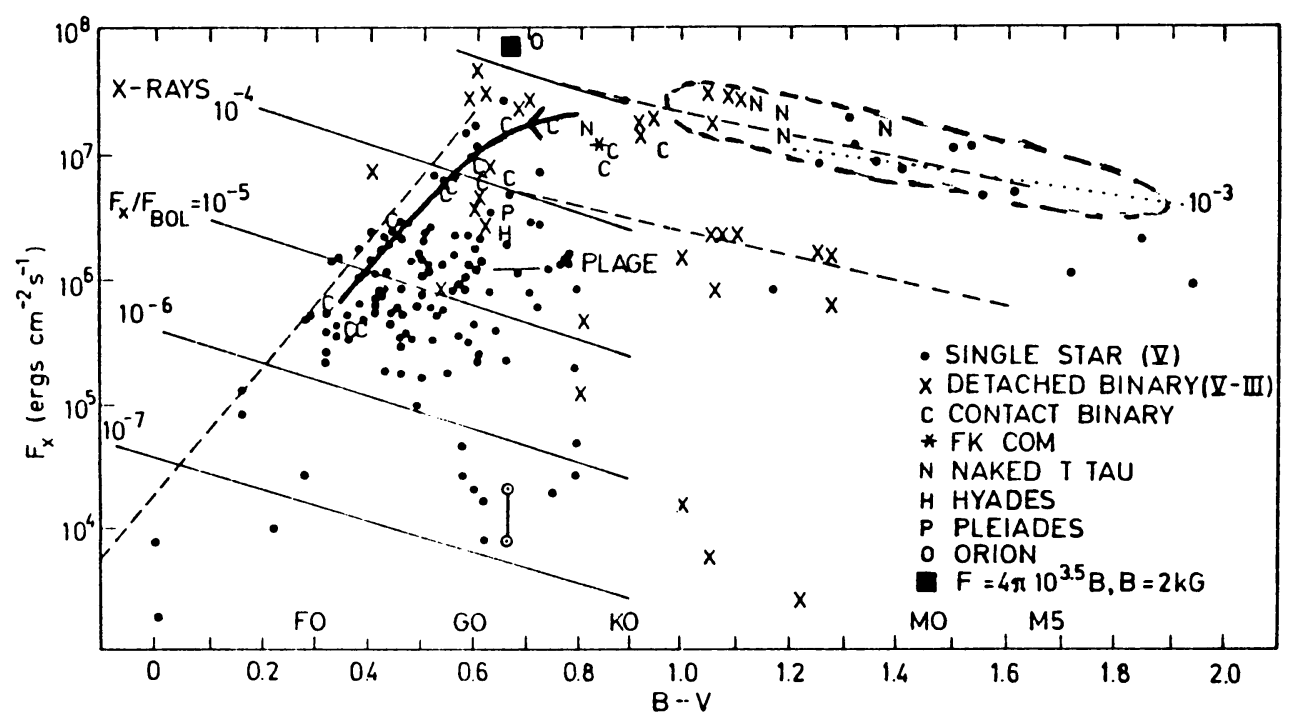

Fig.2 X-ray surface fluxes vs. colour for cool stars of different type. W UMa stars (the solid curve with an arrow) and the cool secondaries of CV's and LMXB's (the dashed oval) are discussed in the text.

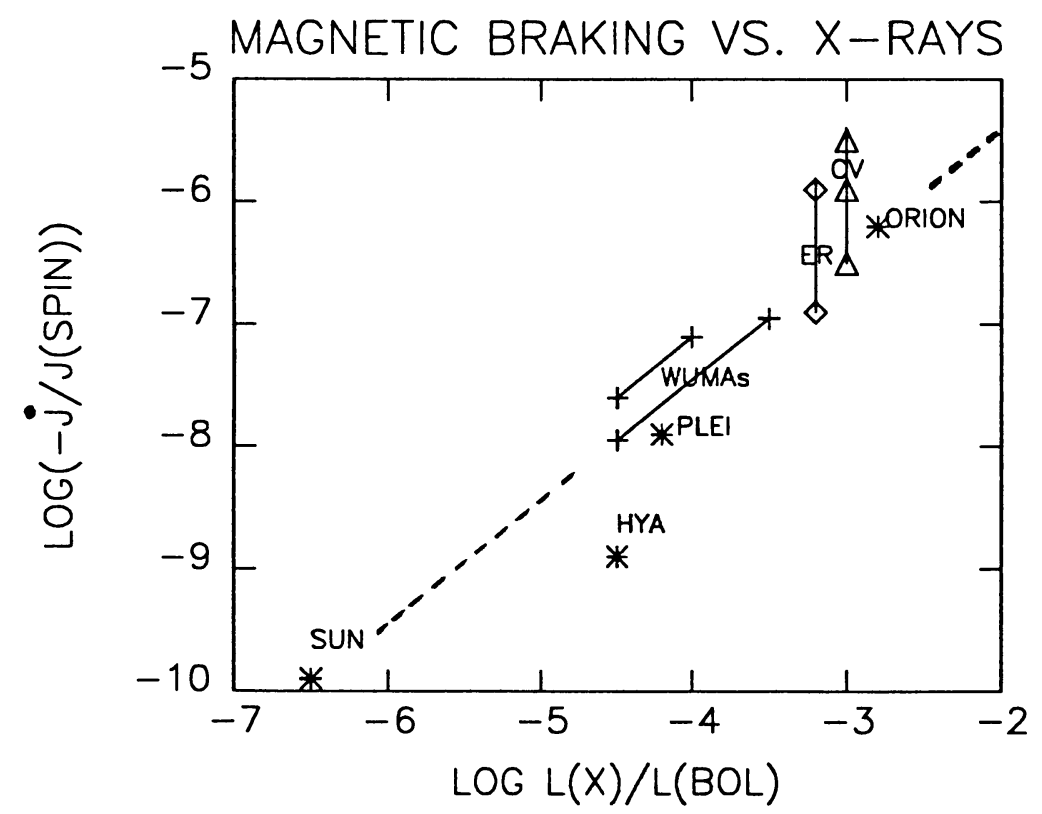

Fig. 3 The semi-empirical relation between the scaled magnetic torque and the $\mathrm{X}$-ray luminosity. The dashed line is eq. (5) (see the text). 

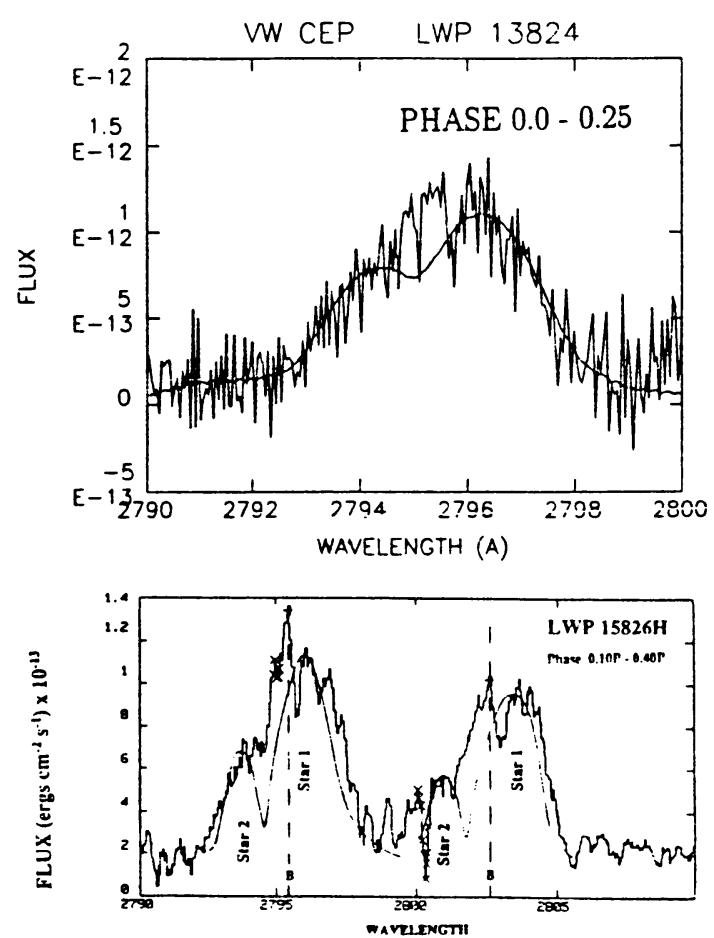

Fig.4 High resolution MgII spectra of VW Cep taken around phase 0.25 with the IUE satellite. The solid curves show the theoretical spectra expected. Note the extra emission between the components. The lower $h+k$ spectrum is from Bradstreet and Guinan (1990), the upper one from the present study.

\subsection{Spots}

Mapping of spots and active regions is an important and growing field in cool star research (for a good review, see Linsky, 1988). Light curves of W UMa stars are asymmetric and variable. These are often interpreted by cool photospheric spots on the primary component (just to mention a few works: Linnell and Olson, 1989; Bell, Rainger and Hilditch, 1990).

A good example is VW Cep (Bradstreet and Guinan, 1990), in which a 10 years spot cycle was resolved. During the minimum phase (1984), the X-ray and transition region (TR) fluxes experienced clear orbital modulation (Vilhu and Heise, 1986 ), but during the spot maxima the X-rays (Cruddace and Dupree, 1984) and the TR fluxes (Bradstreet and Guinan, 1990) did not show any modulation, possibly due to the contribution from active regions connected with the spots. Large changes between different epochs (1982- 1989) have been observed also in 44 Boo (Rucinski and Vilhu, 1983; Vilhu, Neff and Rahunen, 1989; Barstow, Bromage and Holberg, 1990). 
Modelling of photometric ground-based light curves is the most economic way to study spots (see Rodono, 1991). However, all attempts of this type suffer from the uniqueness problem: it is difficult to separate hot and cool spots, and the uniqueness of the contact solution itself makes troubles. Better results can (in principle) be expected if the photometric techniques are combined with the spectroscopic Doppler imaging method.

The Doppler imaging method has been applied to late type stars by Vogt and Penrod (1983), Vogt, Penrod and Hatzes (1987), Piskunov, Tuominen and Vilhu (1990) and Collier Cameron (1991). In the method one introduces additional constraints to choose the 'correct' solution. Normally an entropy regularization function $(R)$ is used, to search for the highest information entropy. One could also use other forms of the regularization function (e.g. to search for the smoothest solution).

The whole problem is to find an extremum of the function $Q=R-L D$, where $D$ is the sum of the differences squared between the observed and computed spectra. The sum is taken over all the observed phases and wavelengths. $L$ is the so called Lagrangian multiplier.

To illustrate the combined photometric and spectroscopic mapping we present some results from Maceroni, Van't Veer and Vilhu (1991). The photometric light curves were modelled with the Wilson-Devinney code allowing surface temperature inhomogeneities. The best fit to the $\mathrm{B}$ and $\mathrm{V}$ light curves is shown in Fig. 5. The equivalent width of the model $\mathrm{H} \alpha$ line was varied along the surface and the best solution found by the maximum entropy method (see Fig.5). The photospheric equivalent width of the line depends on the effective temperature:

$\mathrm{EW}=\mathrm{c} \mathrm{T}_{\text {eff }}{ }^{5.5}$ in the models of Kurucz (1979) around $5500 \mathrm{~K}-6500 \mathrm{~K}$. Hence, the equivalent widths can be converted into a temperature scale if no chromospheric contribution is assumed (Barden (1985) interpreted the difference between the components in $\mathrm{H} \alpha$ as due to an active chromosphere on the primary).

Fig.6 shows the surface images. The photometric and spectroscopic maps look qualitatively similar (in both maps dark means cool and bright means hot), although some differences exist. The temperature range of the maps is $5200 \mathrm{~K}-6100 \mathrm{~K}$.

The combined photometric and spectroscopic surface mapping is promising and certainly serves more attention in the future. In particular, bigger telescopes $(4 \mathrm{~m}$ size) should be used to model weaker lines whose cores are not contaminated by possible chromospheres.

\section{Conclusions}

Evolution by angular momentum loss is an attractive scenario to explain the origin and evolution of most (perhaps not all) W UMa stars. According to this picture their progenitors are short period (1-4 d) detached systems and the final products are single stars ( FK Comae type or blue stragglers). 


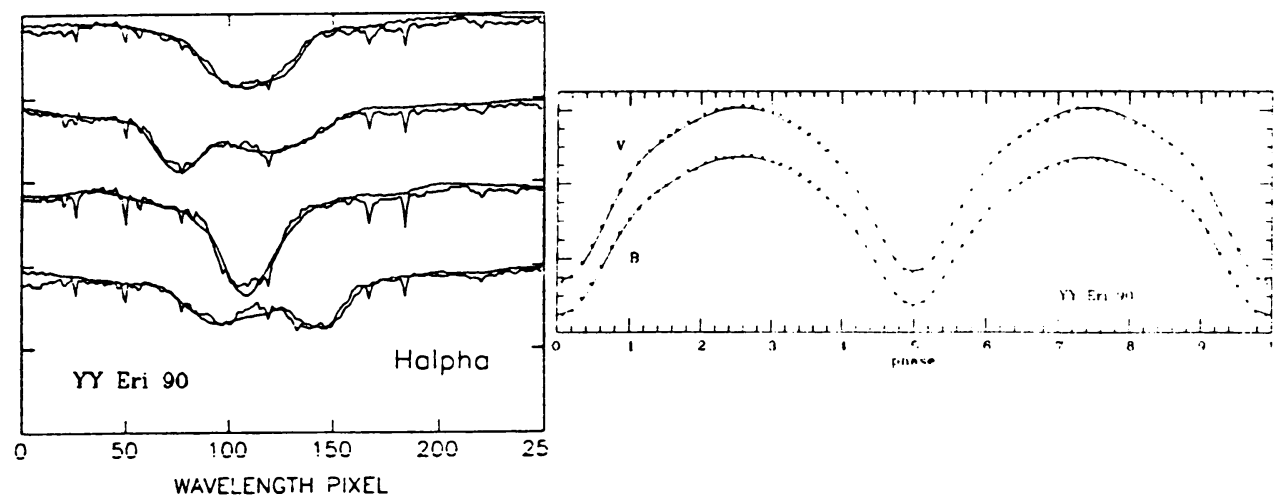

Fig.5 Simultaneous B and V light curves (right) and Ho spectra (left, at phases 0.0 (top), 0.25, 0.5 and 0.75) (from Maceroni et al. 1991).

YY ERI
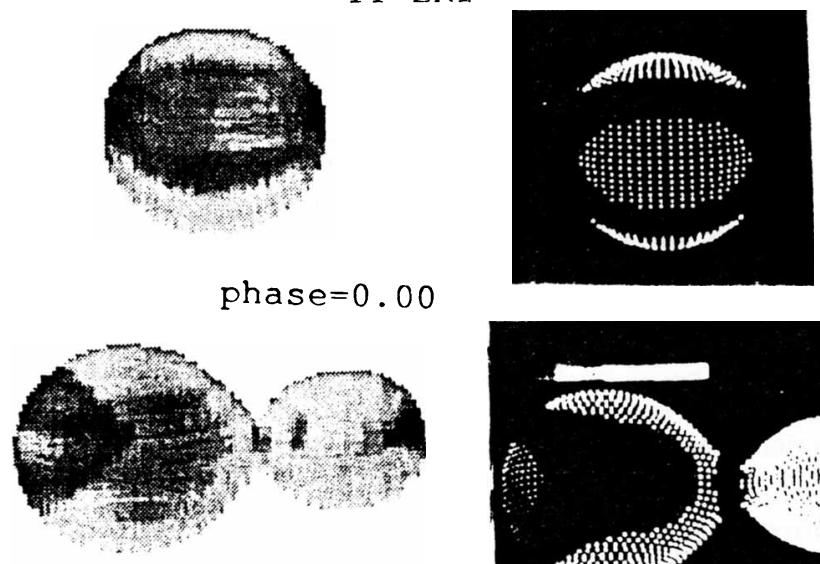

phase $=0.25$
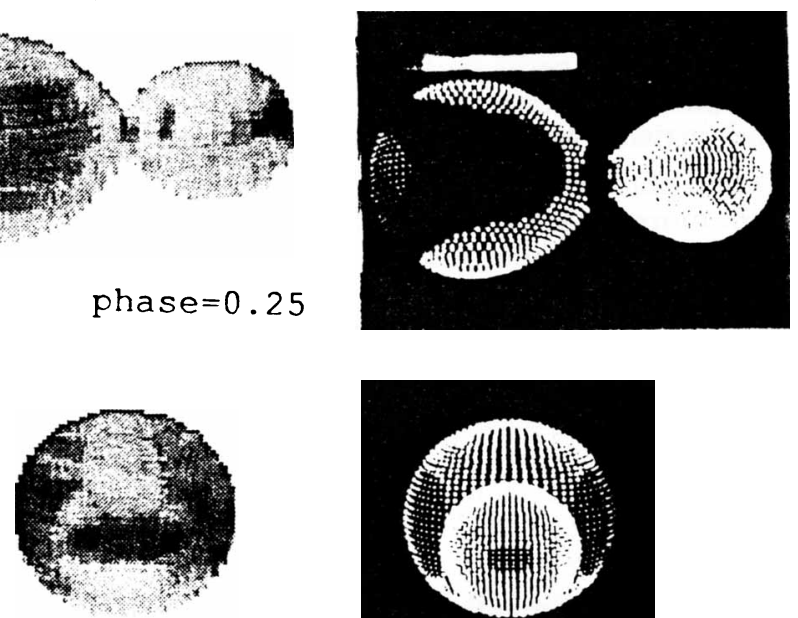

$$
\text { phase }=0.50
$$

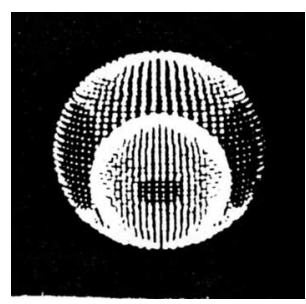

Fig.6 Simultaneous $\mathrm{H} \alpha$ (left) and photometric (right) surface maps of YY Eri. In both maps the temperature range is between $6100 \mathrm{~K}$ (bright) and $5200 \mathrm{~K}$ (dart: (from Maceroni, V'an't Veer and Vilhu, 1991). 
For short periods (less than 3 days), the parameter $\alpha$ in the braking law (1) should be around 1.5. The observationally justified value of $\gamma$ is 2 , and the $f$ parameter should be computed from eq.(2). These parameter values should be valid for short period detached and contact binaries, and for the cool components of CV's and LMXB's. A careful study of the O-C diagrams could (in principle) be a crucial test of these values and the whole braking law.

For different types of stars there seems to be a semi-empirical relation between the scaled magnetic torques and the X-ray luminosities (via eq. (5)). In Mestel's (1968) type theories this can be qualitatively understood, but quantitatively it remains to be explained.

The theory predicts more magnetic activity on the primary component. In many cases the observations are fitted by an active primary (spots, active regions) but this does not need to be the final truth. Surface imaging with simultaneous photometry and high resolution spectroscopy should be the method of future, to follow the evolution of spots and perhaps to see even the neck (see Ch.3).

\section{references}

Barden, S.: 1985, Astrophys. J. 295, 162.

Barstow, M.A., Bromage, G.E. and Holberg, J.B.: 1990, in Evolution in Astrophysics, ESA SP-310, p. 375.

Bell, S.A., Rainger, P.P., Hilditch, R.W.: 1990, Mon.Not.R.astr.Soc. 247, 632.

Bopp, B.W. and Rucinski, S.M.: 1981, in D. Sugimoto, D.Q. Lamb, D.N. Schramm (eds.) Late Stages of Stellar Evolution, D. Reidel Publ. Co., p.181.

Budding, E., Slee O.B., Stewart, R.T., Beasley, A.J., Ball, L.: 1991, these proceedings.

Bradstreet, D.H. and Guinan, E.F.: 1990, in C. Ibanoglu (ed.) Active Close Binaries, Kluwer Academic Publishers, p. 467.

Caillault,J.-P. and Helfand : 1985, Astrophys. J. 289, 279.

Catalano, S., Marilli, E., Trigilio, C.: 1988, in A.K. Dupree and M.T.V.T. Lago (eds.) Formation and Evolution of Low Mass Stars, Kluwer Academic Publishers, p. 377.

Collier Cameron, A.: 1991, in Surface Inhomogeneities on Late Type Stars, Lecture Notes in Physics, Springer Verlag (in press).

Cruddace, R.G. and Dupree, A.K.; 1984, Astrophys. J. 277, 263.

Eggen, O.J. and Iben, I.: 1990, Astron. J. 97, 431.

Fleming, T.A., Gioia, I.M., Maccacaro, T.: 1989, Astron. J. 98, 692.

Guinan, E.F. and Bradstreet, D.H.: 1988, in A.K. Dupree and M.T.V.T. Lago (eds.) Formation and Evolution of Low Mass Stars, Kluwer Academic Publishers, p.345.

Herczeg, G.: 1991, these proceedings.

Hilditch, R.W., King, D.J. and McFarlane, T.M.: 1988, Mon.Not.R.astr.Soc. 231, 341.

Huang, S.-S.: 1966, Astrophys. J.29, 331.

Kim, C.-H.: 1991, these proceedings.

Kurucz, R.: 1979, Astrophys. J. Suppl. 40, 1. 
Linnell, A.P. and Olson, E.C.: 1989, Astrophys. J. 343, 909.

Linsky, J.L.:1988, in F.A. Cordova (ed.) Multiwavelength Astrophysics, Cambridge Univ. Press, p.49.

Maceroni, C., Van't Veer, F. and Vilhu, O.: 1991, in preparation.

Mateo, M., Harris, H.C., Nemec, J., Olszew'ski, E.W.: 1990, Astron. J. 100, 469.

Mestel, L.: 1968, Mon.Not.R.astr.Soc.138, 359.

Mestel, L. and Spruit,H.C.: 1987, Mon.Not.R.astr.Soc 226, 57.

Moss, D.: 1986, Mon.Not.R.astr.Soc. 218, 247.

Pallavicini, R., Golub, L., Rosner, R., Vaiana, G.S., Ayres, T. and Linsky, J.L.: 1981, Astrophys. J. 248, 279.

Piscounov, N.E., Tuominen, I. and Vilhu, O.: 1990, Astron. Astrophys. 230, 363.

Rahunen, T.: 1981, Astron. Astrophys. 102, 81.

Rahunen, T. and Vilhu, O.: 1982, in Z. Kopal and J. Rahe (eds.) Binary and Multiple Stars as Tracers of Stellar Evolution, D. Reidel Publ. Co., p. 289.

Rappaport, S., Verbunt, F. and Joss, P.C.: 1983 Astrophys. J. 275, 713.

Rodono, M.: 1991, these proceedings.

Rucinski, S.M.: 1985a, in P.P. Eggleton and J.E. Pringle (eds.) Interacting Binary Stars, D. Reidel Publ. Co., p.85.

Rucinski, S.M.: 1985b, in P.P. Eggleton and J.E. Pringle (eds.) Interacting Binary Stars, D. Reidel Publ. Co., p. 113.

Rucinski, S.M.: 1989, Comments Astrophys. 14, 79.

Rucinski, S.M. and Vilhu, O.: 1983, Mon.Not.R.astr.Soc 202, 1221.

Schatzman, E.: 1962, Ann.Astrophys.25, 18.

Skumanich, A.: 1972, Astrophys. J. 171, 565.

Verbunt, F. and Zwaan, C.: 1981, Astron. Astrophys. Lett. 100, L7.

Van't Veer, F.: 1975, Astron. Astrophys. 40, 167.

Van't Veer, F.: 1979, Astron. Astrophys. 80, 287.

Van't Veer, F. and Maceroni, C.: 1989, Astron.Astrophys., 220, 128.

Vilhu, O.: 1982, Astron. Astrophys. 109, 17.

Vilhu, O. and Heise, J.: 1986, Astrophys.J. 311, 937.

Vilhu, O. and Moss, D.: 1986, Astron. J. 92, 1178.

Vilhu, O.: 1987, in J.L. Linsky and R.E. Stencel (eds.) Cool Stars, Stellar Systems and the Sun, Springer Verlag, p. 110.

Vilhu, O. and Walter, F.M.: 1987, Astrophys. J. 321, 958.

Vilhu, O., Neff, J.E. and Rahunen, T.: 1989, Astron. Astrophys. 208, 201.

Webbink, R.F.: 1976, Astrophys. J. 209, 829.

Vogt, S.S. and Penrod, G.D.: 1983, Publ.Astron.Soc.Pac. 95, 565.

Vogt, S.S., Penrod, G.D. and Hatzes, A.P.: 1987, Astrophys. J. 321, 496.

Yamasaki, A.: 1975, Aph.Sp.Sci 34, 413.

\section{Acknowledgements}

I am grateful to Carla Maceroni for the pleasant collaboration and for the unpublished work on YY Eri, to Nick Piscounov for the conjugate gradient algorithm and to Thomas Hackman for help in plotting. I warmly thank the organizers of the Symposium for the good and stimulating meeting. 